\title{
Predicting the functional impact of residual aortic coarctation lesions during exercise using advanced computer model simulations.
}

\author{
$\underline{\text { L. Taelman }}{ }^{\mathrm{a}}$, J. Bols ${ }^{\mathrm{b}}$, J. Degroote ${ }^{\mathrm{b}}$, V. Muthurangu ${ }^{\mathrm{c}}$, J. Panzer ${ }^{\mathrm{d}}$, A. Swillens ${ }^{\mathrm{a}}$, J. \\ Vierendeels ${ }^{\mathrm{b}}$, P. Segers ${ }^{\mathrm{a}}$ \\ aIBiTech-bioMMeda - Ghent University, De Pintelaan 185, 9000 Ghent, Belgium; \\ ${ }^{\mathrm{b}}$ Department of Flow, Heat and Combustion Mechanics - Ghent University, Sint- \\ Pietersnieuwstraat 41, 9000 Ghent, Belgium \\ ${ }^{c}$ Centre for Cardiovascular MR, UCL Institute of Child Health, London, United Kingdom \\ ${ }^{\mathrm{d}}$ Paediatric Cardiology, University Hospital of Ghent, Ghent, Belgium
}

Background Exercise can be used to unmask the functional impact of a residual narrowing and/or stiffening following treatment of aortic coarctation. Measurements of the hemodynamic response during exercise are, however, difficult to perform and not very accurate, as suspension of exercise during imaging can be required or a low temporal resolution might be insufficient at the high heart rates present during exercise. This work aims to predict central aortic hemodynamics during exercise using advanced modeling tools.

Material and methods The geometry and the flow boundary conditions, used in this model, are obtained from MRI data at rest (Figure 1). As model simulations with rigid walls fail to capture important physiological phenomena such as wave propagation and reflections, the fluid-structure interaction between the blood flow and the deformation of the arterial wall is taken into account.

Results The numerical model is first calibrated to the resting conditions such that the predicted aortic distensions match the measured values. Predicted velocities throughout the aorta are compared to $4 \mathrm{D}$ velocity measurements. Next, exercise conditions are simulated, with blood flows obtained using an MRI-compatible bycicle type ergometer, in which motion is restricted to the lower legs. Model output includes pressure along the aorta and velocity fields during exercise conditions.

Conclusions. Advanced modelling techniques allow to simulate aortic hemodynamics in $3 \mathrm{D}$ and are capable to provide complementary data that are difficult to obtain in vivo.

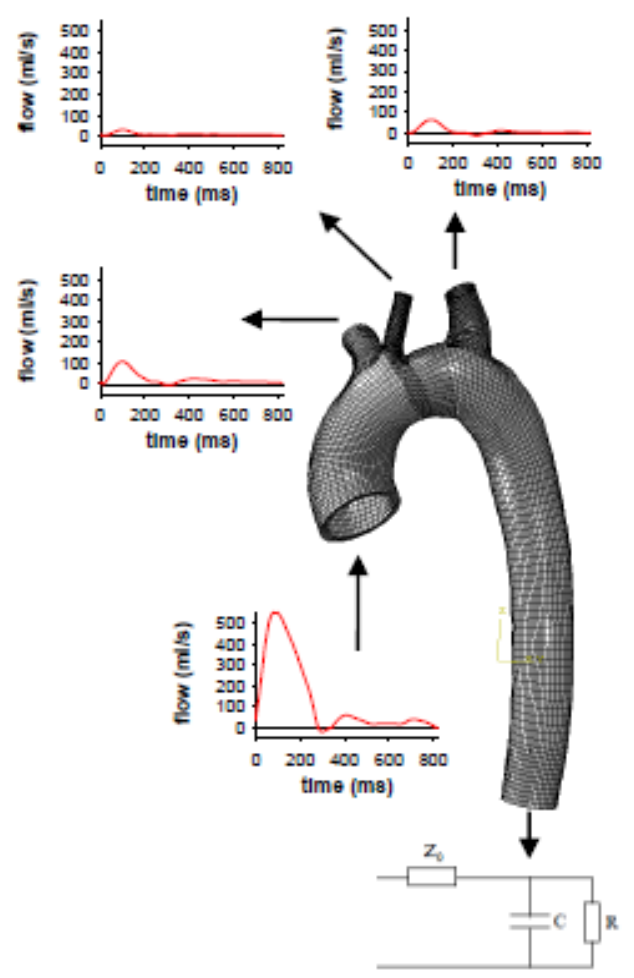

Figure 1. Patient-specific geometry and boundary conditions. 